\title{
Intercostal Cryo Nerve Block in Minimally Invasive Cardiac Surgery: The Prospective Randomized FROST Trial
}

\author{
Wei C. Lau (D) · Francis L. Shannon · Steven F. Bolling • \\ Matthew A. Romano · Marc P. Sakwa · Andrea Trescot • \\ Luoxi Shi · Robert L. Johnson • Vaughn A. Starnes · John F. Grehan
}

Received: August 3, 2021 / Accepted: September 2, 2021 / Published online: September 20, 2021

(C) The Author(s) 2021

\begin{abstract}
Introduction: Intercostal cryo nerve block has been shown to enhance pulmonary function recovery and pain management in post-thoracotomy procedures. However, its benefit have never been demonstrated in minimal invasive thoracotomy heart valve surgery (Mini-HVS). The purpose of the study was to determine whether intraoperative intercostal cryo nerve block in conjunction with standard of care (collectively referred to hereafter as CryoNB) provided superior analgesic
\end{abstract}

W. C. Lau $(\varangle) \cdot$ F. L. Shannon · R. L. Johnson William Beaumont Hospital, 3601 West 13 Mile Road, Royal Oak, MI 48703, USA

e-mail: Wei.lau@beaumont.edu

S. F. Bolling · M. A. Romano

University of Michigan, Ann Arbor, MI, USA

M. P. Sakwa

Memorial Care Heart and Vascular Institute, Long Beach, CA, USA

A. Trescot

The Pain and Headache Center, Eagle River, AK, USA

L. Shi

NAMSA, Minneapolis, MN, USA

V. A. Starnes

University of Southern California, Los Angeles, CA, USA

J. F. Grehan

United Heart and Vascular Institute-Allina, Saint

Paul, MN, USA efficacy in patients undergoing Mini-HVS compared to standard-of-care (SOC).

Methods: FROST was a prospective, 3:1 randomized (CryoNB vs. SOC), multicenter trial in patients undergoing Mini-HVS. The primary endpoint was the 48-h postoperative forced expiratory volume in $1 \mathrm{~s}$ (FEV1) result. Secondary endpoints were visual analog scale (VAS) scores for pain at the surgical site and general pain, intensive care unit and hospital length-ofstay, total opioid consumption, and allodynia at 6 months postoperatively.

Results: A total of 84 patients were randomized to the two arms of the trial CryoNB $(n=65)$ and SOC $(n=19)$. Baseline Society of Thoracic Surgeons Predictive Risk of Mortality (STS PROM) score, ejection fraction, and FEV1 were similar between cohorts. A higher 48 -h postoperative FEV1 result was demonstrated in the CryoNB cohort versus the SOC cohort $(1.20 \pm 0.46$ vs. $0.93 \pm 0.43 \mathrm{~L} ; P=0.02$, one-sided two-sample $t$ test). Surgical site VAS scores were similar between the CryoNB and SOC cohorts at all postoperative timepoints evaluated, but VAS scores not related to the surgical site were lower in the SOC group at 72,94 , and $120 \mathrm{~h}$ postoperatively. The SOC cohort had a 13\% higher opioid consumption than the CryoNB cohort. One of 64 CryoNB patients reported allodynia that did not require pain medication at 10 months.

Conclusions: The results of FROST demonstrated that intercostal CryoNB provided enhanced FEV1 score at $48 \mathrm{~h}$ postoperatively 
with optimized analgesic effectiveness versus SOC. Future larger prospective randomized trials are warranted to determine whether intercostal CryoNB has an opioid-sparing effect in patients undergoing Mini-HVS.

Trial Registration: Clinicaltrials.gov identifier: NCT02922153.

Keywords: Cryo nerve block; Cryoanalgesia; Pain management; Opioids; Analgesia; CryoICE

\section{Key Summary Points}

\section{Why carry out this study?}

Intercostal cryoanalgesia has been shown to manage postoperative pain and improve pulmonary function recovery after full thoracotomy procedures but has not been evaluated in minimally invasive cardiac surgery.

The prospective, randomized FROST study was aimed at assessing whether intercostal cryo nerve block in conjunction with standard-of-care (referred to here as CryoNB) pain management provided superior forced expiratory volume in $1 \mathrm{~s}$ (FEV1) results, recovery, and analgesic efficacy compared to standard of care (SOC) alone in minimally invasive heart valve procedures.

\section{What was learned from the study?}

FEV1 results were significantly improved at $48 \mathrm{~h}$ postoperatively in the CryoNB cohort compared to the cohort receiving SOC alone. While similar effective pain management was observed at the surgical incision site in both cohorts, a more precise therapeutic pain management was observed for general pain in the CryoNB cohort.

Allodynia in the CryoNB arm was low $(1.6 \%)$, and there was a trend of reduced opioid consumption in the CryoNB arm compared to the SOC arm.

The FROST trial also identified important considerations for future study design, including timing of pulmonary function tests and pain assessments.

\section{INTRODUCTION}

Thoracotomy is associated with severe postoperative pain that can result in respiratory complications, such as hypoventilation, hypoxia, atelectasis, pulmonary infections, and respiratory failure $[1,2]$. Postoperative forced expiratory volume in $1 \mathrm{~s}$ (FEV1) is used to define functional operability in patients undergoing thoracotomy and lung resection. A low FEV1 result correlates with postoperative morbidity and mortality as a single predictor [3] or in combination with other variables [4]. Anterior mini-thoracotomy has been advocated as an alternative approach to minimize invasiveness for patients undergoing heart valve surgery (Mini-HVS) requiring cardiopulmonary bypass (CPB) [5]. Optimal post-thoracotomy analgesia can improve pulmonary function and prevent potential complications $[6,7]$.

Treatment of post-thoracotomy pain includes the use of intravenous (IV) opioids, which have limitations due to side effects of sedation and respiratory depression [7, 8]. Intercostal nerve blocks using local anesthetics are effective, but limited by their short duration of action and potential neurotoxicity in high volume $[9,10]$. Epidural analgesia is commonly used for post-thoracotomy without $\mathrm{CPB}$ $[11,12]$. Mini-HVS requires anticoagulation for $\mathrm{CPB}$; consequently, epidural analgesia poses a significant risk for epidural hematoma. Thoracic paravertebral block (TPVB) and interfascial plane blocks of the chest wall, such as erector spinae plane (ESP) block, are alternative treatments for post-thoracotomy pain. However, TPVB is limited in the immediate post-cardiac surgery patient due to the need for optimal prone position and precise ultrasound location of the anatomical landmarks for the injection of local anesthetic. In addition, TPVB is a deep block, performed in noncompressible space, and thus poses a significant risk for paravertebral hematoma due to the tenuous coagulopathic state of the immediate post-cardiac surgical patient [13]. Erector spinae plane block placed at T3-T4 has been reported as a means to manage acute post-surgical pain in adult cardiac procedures [14]. However, it is also limited by 
the need for sitting, lateral decubitus, or prone positioning. Additional risks include local anesthetic systemic toxicity, injury to the long thoracic, or thoracodorsal nerve, epidural spread of local anesthetic, and anticoagulation risks similar to TPVB.

Intercostal cryoanalgesia has been shown to be effective for postoperative pain management for intrathoracic procedures requiring full thoracotomy [15-17], and has resulted in reduced opioid requirements, reduced postoperative visual analogue pain scores (VAS), and superior FEV1 result recovery $[16,17]$. Higher postoperative FEV1 results may correlate to earlier rehabilitation and better outcomes [4]. It is currently unknown whether intercostal cryo nerve block in conjunction with standard of care is superior for pulmonary function recovery (FEV1 results) and pain reduction (VAS) in patients undergoing Mini-HVS requiring $\mathrm{CPB}$ as compared to institutional standard of care (SOC) using opioids [7]. The objective of this study was to determine whether intraoperative intercostal cryo nerve block in conjunction with standard of care (collectively referred to hereafter as CryoNB) provided superior FEV1 results and analgesic efficacy in patients undergoing unilateral thoracotomy cardiac procedures compared to current intraoperative SOC alone.

\section{METHODS}

\section{Patient Population}

FROST (the cryoICE ${ }^{\mathrm{TM}}$ study For pain management in post thoRacic procedures via intercOSTal cryoanalgesia) was a prospective, randomized, multicenter trial (clinicaltrials.gov: NCT02922153). The study was performed in accordance with the Helsinki Declaration of 1964 and subsequent amendments. Institutional review board (IRB) approval and written informed consent were obtained at all centers [University of Michigan, Ann Arbor, MI. (\#HUM00109220), University of Michigan Medical School IRB; Beaumont Health System, Royal Oak, MI (\#2015-438), Beaumont Research Institute IRB; United Heart and Vascular Institute-Allina, Saint Paul, MN (\#32157/1), Quorum
IRB; and University of Southern California, Los Angeles, CA, University of Southern California Health Sciences Campus (IRB; \#HS-17-00066), University of Southern California Health Sciences Campus IRB]. Inclusion criteria were patients $18-85$ years of age undergoing MiniHVS alone or in combination with additional cardiac procedures (Table 4). Exclusion criteria were cardiac surgery via full sternotomy; pregnancy; current use of opioids; FEV1 result $<$ $40 \%$ of the predicted result; myocardial infarction within 30 days of informed consent; history of substance abuse; chronic pain syndrome; and psychiatric, physical, or mental conditions that would interfere with pain assessment. Patients were randomized $3: 1$ to receive intercostal cryoNB using the cryoICE ${ }^{\mathrm{TM}}$ cryoablation probe (AtriCure, Inc., Mason, $\mathrm{OH}$, USA) plus SOC post-operative pain management (the CryoNB cohort) or SOC post-operative pain management alone (SOC cohort). Randomization sequences were generated using SAS version 9.4 (SAS Institute Inc., Cary, NC, USA) by the study sponsor statistician and were stratified by site. Upon enrollment, patients were assigned a sequential identification number at each site and a corresponding sealed envelope that was opened prior to the procedure to reveal the treatment group. Patients and physicians were not blinded to treatment group; however, assessors of FEV1 results and pain were blinded to the assigned group. The first patient was treated in June 2016 and final patient follow-up was completed in August 2019.

\section{Protocol for Intraoperative Intercostal CryoNB}

The cryoICE system is a cryoablation probe with a malleable shaft and a retractable handle to control active probe length (Fig. 1a). The cryoprobe was positioned under direct visualization (Fig. 1b), and ablation of the intercostal nerves was performed at the margin of the pleura and innermost intercostal muscle (Fig. 1c), maintaining a distance of at least $2 \mathrm{~cm}$ from dorsal root ganglia or $4 \mathrm{~cm}$ from the spine. One cryoablation at $-50{ }^{\circ} \mathrm{C}$ to $-70{ }^{\circ} \mathrm{C}$ was 
applied for $120 \mathrm{~s}$ to sustain an ablation length of $2-3 \mathrm{~cm}$ at the level of the intercostal incision. Additional cryoablations were then performed at each level for two levels above and two levels below the level of the incision site, avoiding ablations above the third and below the ninth intercostal spaces (Fig. 1d). The probe was removed after the completion of defrosting to avoid mechanical damage to the intercostal nerve. The exact timing of intraoperative cryo application was not specified by the study protocol and was at the discretion of the attending cardiac surgeon but is usually performed at the time of surgical closure.

\section{Postoperative Pain Management}

In both study arms, standardized IV and oral opioids were used for pain management during the postoperative period as part of institutional SOC. Intercostal nerve block with local anesthetic ( $0.5 \%$ ropivacaine) [10] was used in one center in the SOC cohort, but not in the CryoNB cohort. Study patients from all investigational sites were followed for $48 \mathrm{~h}$ after

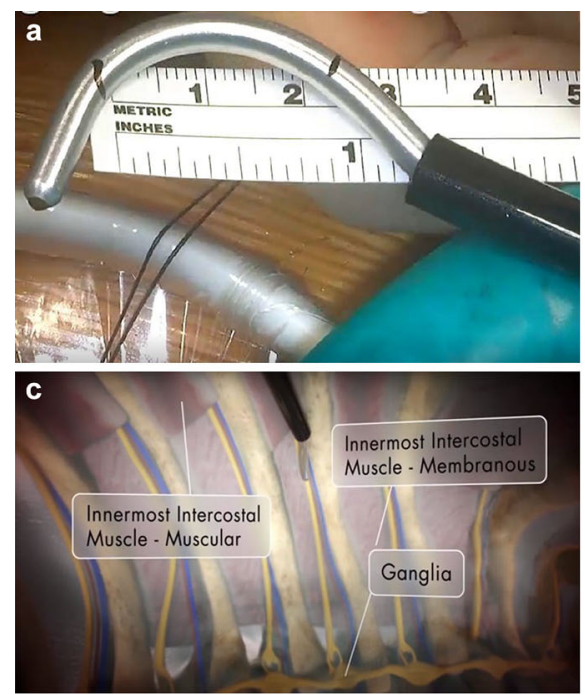

Fig. 1 Description of cryoablation of intercostal nerve. a cryoICE probe bend. $\mathbf{b}$ External view of the position and application of the cryoICE probe. c Internal view of the position of cryoICE probe: ablation of the margin of the membranous section of the intercostal muscle, maintaining a distance of at least $2 \mathrm{~cm}$ from dorsal root ganglia, or $4 \mathrm{~cm}$ surgery, then every $24 \mathrm{~h}$ for $120 \mathrm{~h}$, at hospital discharge, then at 30, 90, and 180 days after discharge. All patients were contacted at 3 and 6 months after surgery by phone to screen for hyperalgesia using a questionnaire. If the questions elicited a positive response, the patient was asked to make an office visit for a more thorough allodynia assessment. The cotton tip applicator test was used, which has been validated as a reproducible screening method to identify cutaneous allodynia [18].

\section{Study Endpoints}

The primary endpoint was the 48 -h postoperative FEV1 result. Secondary endpoints were: VAS pain at surgical site at $48 \mathrm{~h}$ postoperatively (VAS values of " 0 " correspond to no pain, " $1-3$ " to mild pain, "4-6" to moderate pain, and "7-10" to severe pain [19]), FEV1 results, and VAS scores at 72,96 , and $120 \mathrm{~h}$ postoperatively; total procedure time; duration of oral endotracheal tube (OETT) intubation; intensive care unit (ICU) and hospital length of stay (LOS); and the total average opioid consumption per day for

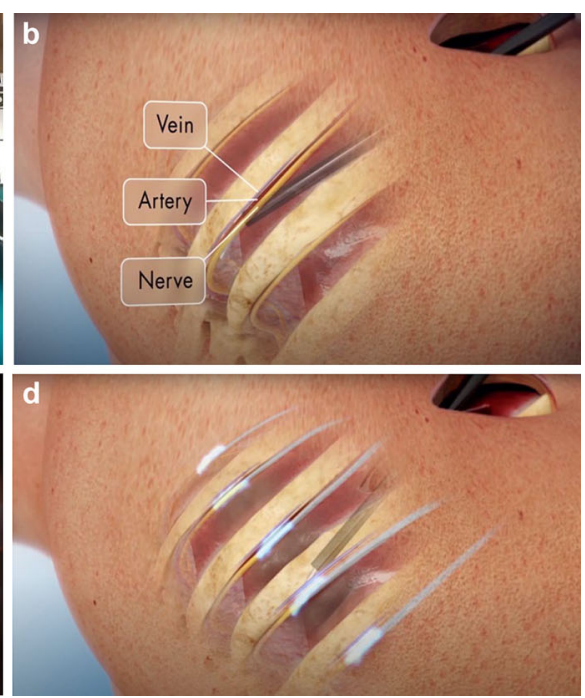

from the base of the probe, maintaining a still and gentle pressure for the duration of cryoablation. $\mathbf{d}$ One cryoablation at the intercostal level of the incision, then ablation two levels above and two levels below the incision site, avoiding going above the third and below the ninth intercostal space 
the entire hospital LOS. All FEV1 were performed using spirometry in a standardized fashion [20], and the results were collected by a single study coordinator at each institution who was blinded to the randomization of the patients in the study. For consistency, spirometry was performed at each Investigational Site at $48,72,96$ and $120 \mathrm{~h}$ postoperatively, using the same spirometry device. Final FEV1 results were an average of three consecutive tests. The VAS based on Mankoski pain scale definitions was used. The patient was asked to complete the scale to rate their current pain level for general pain intensity ("general pain") and to complete the scale for pain intensity related to the surgical region ("surgical site"). Investigational site personnel conducted the test at each site and were blinded to the treatment group of each patient.

\section{Data Collection}

An Electronic Data Capture System was used by trained, designated Investigational site personnel to collect and transfer study data from source records into common eCase Report Forms following a protocol specified and IRB approved visit schedule.

\section{Sample Size Estimation}

Two previous studies of cryoanalgesia on patients undergoing thoracotomy were used to estimate sample size for the study based on FEV1 results [21, 22]. These two studies reported postoperative FEV1 results [mean \pm standard deviation (SD)] at $48 \mathrm{~h}$ of $1.5 \pm 0.7$ and $1.1 \pm 0.47$ with SOC analgesia. Based on this, a FEV1 result of $1.30 \pm 0.39$ was used in our FEV1 result sample size estimation. Based on investigator experience, a 20\% improvement in FEV1 result was considered to be a clinically effective change. Therefore, the expected mean \pm SD of FEV1 for the CryoNB cohort was estimated to be $1.56 \pm 0.39$, assuming a common standard deviation. In effect, with 3:1 randomization, group sample sizes of 75 (cryoNB) and 25 (SOC) would achieve $80 \%$ power to reject the null hypothesis of equal means when the population mean difference is 0.26 with a SD for both groups of 0.39 and with a significance level (alpha) of 0.025 using a one-sided two-sample equal-variance $t$ test. In addition, our preliminary observational study in a series of six patients undergoing single cardiac valve surgery via right mini-thoracotomy reported mean VAS differences between the cryoanalgesia and SOC cohorts to be $1.5(P=0.03)$ and $1.8(P=0.04) 24$ and $48 \mathrm{~h}$ postoperatively, respectively, with a common SD of 2.2. Based on these outcomes, sample sizes of 75 (cryoNB) and 25 (SOC) would achieve $94 \%$ power to reject the null hypothesis of equal means with two-sided alpha of 0.05.

\section{Statistical Analysis}

Summary statistics were presented by treatment group. Continuous variables were summarized by the mean $( \pm S D)$ and median (with minimum, maximum). Categorical variables were summarized as frequencies and percentages [ $n$ (\%)], where $n$ represents the total number of observations in the category and \% represents (observations in the categories/total observation across all categories) $\times 100$. All analyses were performed using SAS version 9.4. Randomization breeches were prevented by the oversight of the Institutional Research Data Safety Monitoring Board. Descriptive statistics were presented for relevant baseline demographics. $P$ values were calculated with the twosample $t$ test or Wilcoxon rank-sum test for continuous variables, and with the Chi-square test or Fisher's exact test for categorical variables. One-sided $P$ value was presented for the primary endpoint. Two-sided $P$ values were presented for secondary endpoints.

\section{Primary and Secondary Endpoints}

The 48-h postoperative FEV1 results were compared between the two treatment groups. Descriptive statistics and the two-sample $t$ test were used to compare FEV1 results between groups at this timepoint. Descriptive statistics were presented for FEV1 results at 72, 96, and $120 \mathrm{~h}$ postoperatively. Descriptive statistics were presented for surgical site and general pain 
VAS scores $48,72,96$, and $120 \mathrm{~h}$ postoperatively; total procedure time; duration of OETT intubation; and ICU and hospital LOS. The Wilcoxon rank-sum test was used for $P$ values. Overall opioid consumption, expressed as morphine milligram equivalents (MME) and divided into oral and IV opioid consumption per day over the duration of hospital LOS, was summarized and compared between groups with the Wilcoxon rank-sum test.

\section{RESULTS}

A total of 84 patients were enrolled, of whom 65 were randomized to the CryoNB arm and 19 to the SOC arm (Fig. 2). Baseline characteristics were similar between the two cohorts. The mean Society of Thoracic Surgeon (STS) Predictive Risk of Mortality (PROM) score and cardiac risk factors were similar between the two cohorts (Table 1). There were no differences in baseline FEV1 results. The baseline VAS was statistically different at the surgical site without distinct clinical implication.

\section{Primary Endpoint}

One patient required conversion from minithoracotomy to full sternotomy related to the index surgical procedure and was withdrawn post-procedurally. Of the remaining 83 patients, 62 patients completed the 48 -h postoperative FEV1 assessment (Table 2). The FEV1 result was higher in the CryoNB cohort than in the SOC cohort $(1.20 \pm 0.46$ vs. $0.93 \pm 0.43 \mathrm{~L}$; $P=0.02$ by one-sided two sample $t$ test) at $48 \mathrm{~h}$ postoperatively.

\section{Secondary Endpoints}

The postoperative FEV1 results at 72,96 , and $120 \mathrm{~h}$ were similar between the cryoNB and SOC groups (Fig. 3). A total of 95\% (79/83) of the patients had completed the VAS assessments at $48 \mathrm{~h}$ postoperatively (Table 3 ). At $48 \mathrm{~h}$ postoperatively, mean VAS pain scores at the surgical site were similar between the CryoNB $(2.82 \pm 2.70)$ and SOC $(3.06 \pm 2.82)(P=0.70)$

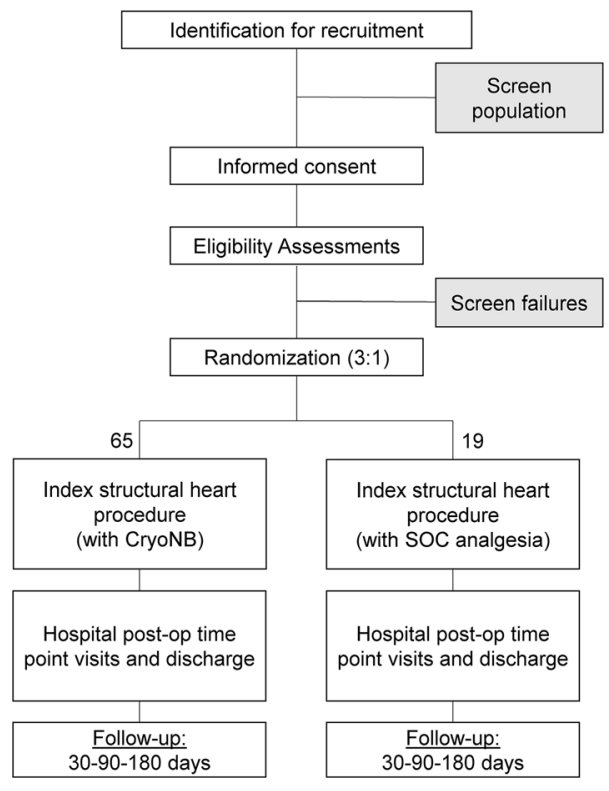

Fig. 2 Overall study and design workflow for the FROST trial

cohorts, and they were also similar at 72, 96, and $120 \mathrm{~h}$ postoperatively (Table 3 ). Mean VAS scores for general pain were significantly lower in the SOC cohort than in the CryoNB cohort at $72 \mathrm{~h}(0.72 \pm 1.53$ vs. $2.25 \pm 2.75$, respectively; $P=0.02), 96 \mathrm{~h}(0.73 \pm 1.79$ vs. $2.20 \pm 2.50$, respectively; $P=0.01)$, and $120 \mathrm{~h}(0.30 \pm 0.95$ vs. $2.14 \pm 2.57$, respectively; $P=0.02$ ) (Table 3 ). The mean operating room (OR) time, duration of OETT intubation, ICU and hospital LOS were similar between the CryoNB and SOC cohorts (Table 4). There were no documented complications of phrenic nerve injury, major vascular injuries requiring intervention, or any injury related to intercostal CryoNB. There were nine mortalities during the study, including three within the first 30 postoperative days and six after the first 30 postoperative days. None were related to the CryoNB.

Without a standardized protocol for administering a minimum effective dose of opioids [23] for postoperative analgesia based on VAS, institutional standards were used for opioid administration. Although the $P$ value from the Wilcoxon rank-sum test was not statistically significant, there was an overall 13\% reduction in the average total opioid consumption (morphine milligram equivalents [24]) during the 
Table 1 Baseline clinical characteristics by cryo nerve block and standard of care

\begin{tabular}{llll}
\hline Variables $^{\mathrm{a}}$ & CryoNB $(\boldsymbol{N}=\mathbf{6 5})$ & SOC $(\boldsymbol{N}=\mathbf{1 9})$ & Two-sided $\boldsymbol{P}$ value \\
\hline Age (year) & $63.83 \pm 11.96$ & $66.42 \pm 12.53$ & $0.41^{\mathrm{d}}$ \\
Female & $27(42 \%)$ & $8(42 \%)$ & $0.96^{\mathrm{e}}$ \\
Male & $38(58 \%)$ & $11(58 \%)$ & $0.96^{\mathrm{e}}$ \\
BMI & $28.61 \pm 5.03$ & $29.95 \pm 4.73$ & $0.30^{\mathrm{d}}$ \\
FEV1 (L) & $2.33 \pm 0.78$ & $2.28 \pm 0.84$ & $0.83^{\mathrm{d}}$ \\
FVC (L) & $3.08 \pm 1.03$ & $3.02 \pm 1.02$ & $0.84^{\mathrm{d}}$ \\
STS PROM score & & $0.37^{\mathrm{f}}$ \\
Atrial fibrillation & $2.03 \pm 1.90$ & $2.86 \pm 2.57$ & $0.98^{\mathrm{e}}$ \\
Congestive heart failure & $31(48 \%)$ & $9(47 \%)$ & $0.80^{\mathrm{e}}$ \\
COPD & $22(34 \%)$ & $7(37 \%)$ & $\mathrm{NA}$ \\
Renal disease & $0(0 \%)$ & $0(0 \%)$ & $\mathrm{NA}$ \\
LVEF (\%) & $0(0 \%)$ & $0(0 \%)$ & $1.00^{\mathrm{f}}$ \\
Stroke & $54.40 \pm 11.37$ & $56.37 \pm 8.64$ & $1.00^{\mathrm{g}}$ \\
Myocardial Infarction & $1(2 \%)$ & $0(0 \%)$ & $0.42^{\mathrm{g}}$ \\
VAS pain score, general & $6(9 \%)$ & $3(16 \%)$ & $0.40^{\mathrm{f}}$ \\
VAS pain score, surgical site & $0.32 \pm 0.95$ & $0.42 \pm 0.90$ & $0.01^{\mathrm{f}}$ \\
\hline BMIB & $0.03 \pm 0.25$ & $0.21 \pm 0.54$ &
\end{tabular}

$B M I$ Body mass index, COPD chronic obstructive pulmonary disease, CryoNB Cryonerveblock, $L V E F$ left ventricular ejection fraction, $F E V I$ forced expiratory volume 1-s, $F V C$ forced vital capacity, $N A$ data not available, $S O C$ standard-ofcare, STS PROM Society of Thoracic Surgeons Predictive Risk of Mortality, VAS visual analog scale

${ }^{a}$ Categorical variables are presented as a number $(n)$ with the percentage in parentheses $[n(\%)]$; continuous variables are presented as the mean \pm standard deviation $(\mathrm{SD})$

${ }^{b}$ Number of subjects for whom a STS PROM score was available in the CryoNB cohort was 60

${ }^{c}$ Number of subjects for whom $\operatorname{LVEF}(\%)$ was available in the CryoNB was 62

${ }^{d} P$ values were calculated with the two-sample $t$ test

e $P$ values were calculated with the Chi-square test

${ }^{f} P$ values were calculated with the Wilcoxon rank-sum test for continuous variables

${ }^{g} P$ values were calculated with the Fisher's exact test for categorical variables

Table 2 FEV1 results at 48 h postoperatively

\begin{tabular}{llll}
\hline Parameter & \multicolumn{1}{c}{ CryoNB cohort $(\boldsymbol{N}=46)$} & SOC cohort $(\boldsymbol{N}=\mathbf{1 6})$ & One-sided $\boldsymbol{P}$ value \\
\hline 48-h postoperative FEV1 results & & \\
Mean (mean \pm SD) & $1.20 \pm 0.46$ & $0.93 \pm 0.43$ & $0.02^{\mathrm{a}}$ \\
Median $(\min , \max )$ & $1.19(0.41,2.18)$ & $0.88(0.28,1.84)$ & \\
\hline
\end{tabular}

FEV1 Forced expiratory volume in $1 \mathrm{~s}$, max maximum, min minimum

a $P$ value was calculated with the one-sided two-sample $t$ test 


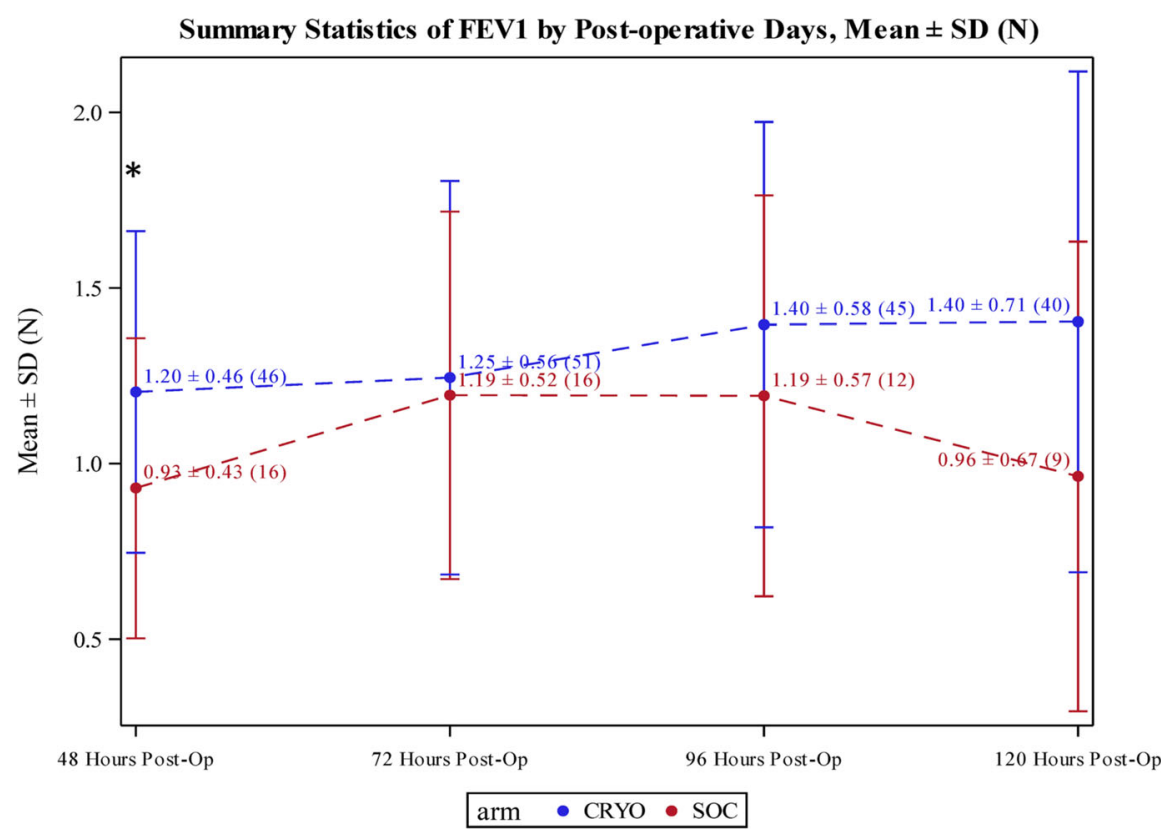

Fig. 3 Summary statistics for FEV1 results by postoperative time (from $48 \mathrm{~h}$ through to $120 \mathrm{~h}$ ) in the CryoNB and SOC cohorts. Results are from two sample $t$ tests and are presented as the mean $\pm \mathrm{SD}(N)$, where $N=$ number of FEV1 samples collected for analysis. The asterisk

hospital LOS in the cryoNB cohort compared to the SOC cohort, which included a $22.7 \%$ reduction in oral opioid consumption and $1.24 \%$ reduction in IV opioid consumption (Table 5). Allodynia screening was done at 3 and 6 months after patient discharge. Of the total, $86 \%(72 / 84)$ of patients completed the 6-month follow-up. Allodynia was reported in one patient $(1.6 \%)$ in the cryoNB cohort, who did not require further intervention through 10 months of follow-up after the positive allodynia test.

\section{DISCUSSION}

FROST was the first trial of intraoperative intercostal CryoNB in patients undergoing mini-HVS requiring $\mathrm{CPB}$. The current study demonstrated that compared with SOC, the addition of intraoperative intercostal CryoNB to SOC resulted in: (1) a higher 48-h postoperative FEV1 result; (2) achieving therapeutic mean VAS scores similar to those achieved with SOC indicates a significant difference between the CryoNB and SOC cohorts at the respective timepoint. CryoNB Intercostal cryo nerve block in conjunction with SOC, FEVI Mean forced expiratory volume in $1 \mathrm{~s}$, SOC standard of care

alone at the surgical site, suggesting comparable pain control [25], and higher therapeutic mean VAS for general pain, suggesting a more optimized analgesic efficacy; (3) a trend for reduced opioid consumption; and (4) a low risk of allodynia in long-term follow-up.

\section{Higher Postoperative FEV1 Result}

FROST demonstrated that intercostal CryoNB provided better 48 -h postoperative FEV1 results in patients who underwent mini-HVS requiring CPB. A trend of better FEV1 result recovery was also observed at 72,96 , and $120 \mathrm{~h}$ postoperatively, but this trend was not significant versus SOC. These results are in line with previously published studies using cryoanalgesia in patients undergoing general thoracic procedures. Moorjani et al. found a trend of improved incentive spirometry results with cryoanalgesia versus conventional analgesia, including FEV1 results during the early post-operative period [16]. Sepsas et al. reported significant 
Table 3 Mean and median VAS pain score at surgical site and for general pain over time by CryoNB and SOC

\begin{tabular}{|c|c|c|c|}
\hline Variables $^{\mathrm{a}}$ & CryoNB cohort & SOC cohort & Two-sided $P$ value ${ }^{b}$ \\
\hline \multicolumn{4}{|c|}{ VAS pain score at surgical site } \\
\hline $48 \mathrm{~h}$ postoperatively & & & 0.70 \\
\hline Mean \pm SD $(n)$ & $2.82 \pm 2.70(61)$ & $3.06 \pm 2.82(18)$ & \\
\hline Median (min, max) & $2.00(0.00,8.00)$ & $2.50(0.00,8.00)$ & \\
\hline $72 \mathrm{~h}$ postoperatively & & & 0.31 \\
\hline Mean $\pm \operatorname{SD}(n)$ & $2.08 \pm 2.39(59)$ & $1.44 \pm 2.09(18)$ & \\
\hline Median (min, max) & $1.00(0.00,7.00)$ & $0.00(0.00,6.00)$ & \\
\hline $96 \mathrm{~h}$ postoperatively & & & 0.82 \\
\hline Mean \pm SD $(n)$ & $1.84 \pm 2.39(51)$ & $2.00 \pm 2.45(15)$ & \\
\hline Median (min, max) & $1.00(0.00,8.00)$ & $1.00(0.00,7.00)$ & \\
\hline $120 \mathrm{~h}$ postoperatively & & & 0.73 \\
\hline Mean $\pm \operatorname{SD}(n)$ & $2.50 \pm 2.82(42)$ & $2.40 \pm 3.37(10)$ & \\
\hline Median (min, max) & $1.00(0.00,9.00)$ & $1.00(0.00,10.00)$ & \\
\hline \multicolumn{4}{|c|}{ VAS pain score for general pain } \\
\hline $48 \mathrm{~h}$ postoperatively & & & 0.75 \\
\hline Mean \pm SD $(n)$ & $3.26 \pm 2.75(61)$ & $3.06 \pm 2.79(17)$ & \\
\hline Median (min, max) & $3.00(0.00,10.00)$ & $3.00(0.00,8.00)$ & \\
\hline $72 \mathrm{~h}$ postoperatively & & & 0.02 \\
\hline Mean $\pm \operatorname{SD}(n)$ & $2.25 \pm 2.75(59)$ & $0.72 \pm 1.53(18)$ & \\
\hline Median (min, max) & $1.00(0.00,10.00)$ & $0.00(0.00,6.00)$ & \\
\hline $96 \mathrm{~h}$ post-op & & & 0.01 \\
\hline Mean $\pm \operatorname{SD}(n)$ & $2.20 \pm 2.50(51)$ & $0.73 \pm 1.79(15)$ & \\
\hline Median (min, max) & $1.00(0.00,8.00)$ & $0.00(0.00,6.00)$ & \\
\hline $120 \mathrm{~h}$ postoperatively & & & 0.02 \\
\hline Mean \pm SD $(n)$ & $2.14 \pm 2.57(42)$ & $0.30 \pm 0.95(10)$ & \\
\hline Median (min, max) & $1.00(0.00,9.00)$ & $0.00(0.00,3.00)$ & \\
\hline
\end{tabular}

${ }^{a}$ Continuous variables are presented as the mean $\pm \mathrm{SD}$ and as the median $(\min , \max )$

b $P$ values were calculated from Wilcoxon rank-sum test for continuous variables

improvements in FEV1 and forced vital capacity results with cryoanalgesia at 30 and 60 days postoperatively compared to IV patient-controlled analgesia [17].

\section{Achieving an Optimized Therapeutic VAS}

The goal of postoperative pain management is not to eliminate all pain [26]. An optimized VAS therapeutic pain target of $2-3$ has been well 
Table 4 Post-procedural results by cryoNB and SOC cohorts

\begin{tabular}{|c|c|c|c|}
\hline Variables & CryoNB cohort $(N=65)$ & SOC cohort $(N=19)$ & Two-sided $P$ value \\
\hline \multicolumn{4}{|l|}{ Cardiac procedures performed ${ }^{a}$} \\
\hline Mitral valve repair/replacement & $54(83 \%)$ & $18(95 \%)$ & $0.28^{\mathrm{d}}$ \\
\hline Tricuspid valve repair/replacement & $16(25 \%)$ & $7(37 \%)$ & $0.29^{c}$ \\
\hline Aortic valve replacement & $2(3 \%)$ & $0(0 \%)$ & $1.00^{\mathrm{d}}$ \\
\hline Atrial septal defect repair & $4(6 \%)$ & $0(0 \%)$ & $0.57^{\mathrm{d}}$ \\
\hline Atrial fibrillation procedure & $27(42 \%)$ & $8(42 \%)$ & $0.96^{\mathrm{c}}$ \\
\hline Valve + atrial fibrillation procedure & $25(38 \%)$ & $7(37 \%)$ & $0.90^{\mathrm{c}}$ \\
\hline Atrial septal defect $+\mathrm{AF}$ procedure & $1(2 \%)$ & $0(0 \%)$ & $1.00^{\mathrm{d}}$ \\
\hline LAA excision or exclusion & $27(42 \%)$ & $10(53 \%)$ & $0.39^{\mathrm{c}}$ \\
\hline Other & $18(28 \%)$ & $3(16 \%)$ & $0.38^{\mathrm{d}}$ \\
\hline \multicolumn{4}{|l|}{ Post-procedural results ${ }^{\mathrm{b}}$} \\
\hline Total OR time $(\mathrm{h})$ & & & $0.52^{\mathrm{e}}$ \\
\hline Mean $\pm \operatorname{SD}(n)$ & $4.76 \pm 1.56(64)$ & $4.51 \pm 1.33(19)$ & \\
\hline Median (min, $\max )$ & $4.32(2.05,8.68)$ & $4.12(2.82,7.45)$ & \\
\hline Time of OETT $(h)$ & & & $0.44^{\mathrm{e}}$ \\
\hline Mean \pm SD $(n)$ & $8.85 \pm 11.16(60)$ & $7.33 \pm 4.51(19)$ & \\
\hline Median $(\min , \max )$ & $4.81(0.00,69.73)$ & $6.85(1.65,17.87)$ & \\
\hline ICU LOS (h) & & & $0.29^{\mathrm{e}}$ \\
\hline Mean \pm SD $(n)$ & $119.03 \pm 186.07(60)$ & $71.07 \pm 53.71(19)$ & \\
\hline Median $(\min , \max )$ & $71.48(13.02,1344.2)$ & $64.35(17.50,236.18)$ & \\
\hline Hospital LOS (days) & & & $0.10^{\mathrm{e}}$ \\
\hline Mean \pm SD $(n)$ & $9.40 \pm 8.34(60)$ & $6.32 \pm 2.56(19)$ & \\
\hline Median $(\min , \max )$ & $6.42(3.25,56.45)$ & $5.32(3.23,12.17)$ & \\
\hline
\end{tabular}

ICU Intensive care unit, $L A A$ left atrial appendage, $L O S$ length of stay, OETT oral endotracheal tube, OR operating room ${ }^{a}$ Categorical variables are presented as $n(\%)$

b Continuous variables are presented as the mean $\pm \mathrm{SD}(n)$ or as the median (min, max)

c $P$ values were calculated with the Chi-square test

d $P$ values were calculated with the Fisher's exact test

${ }^{\text {e }} P$ values were calculated with the Wilcoxon rank-sum test for continuous variables

validated as a minimal effective level needed for the management of postoperative pain $[7,19]$. We have observed that VAS scores for general pain and pain at the surgical incision can differ. As such, we reported both. In our study, CryoNB achieved mean VAS scores of 2-3 that were consistent with an optimized analgesic efficacy $[25,26]$. As a result, CryoNB achieved an analgesic VAS target with less opioid consumption. In contrast, the SOC cohort consistently 
Table 5 Opioid consumption during hospital length of stay by CryoNB and SOC

\begin{tabular}{llll}
\hline Variables $^{\mathbf{a}}$ & $\begin{array}{l}\text { CryoNB cohort } \\
(\boldsymbol{N}=\mathbf{6 3})\end{array}$ & $\begin{array}{l}\text { SOC cohort } \\
(\boldsymbol{N}=\mathbf{1 9})\end{array}$ & $\begin{array}{l}\text { Reduction in opioid consumption in } \\
\text { the CryoNB cohort (\%) }\end{array}$ \\
\hline $\begin{array}{l}\text { Opioid consumption (MME) per day for } \\
\text { entire hospital LOS }\end{array}$ & $33.43 \pm 29.77$ & $38.31 \pm 31.05$ & 12.7 \\
$\begin{array}{l}\text { Oral opioid consumption } \\
\text { IV opioid consumption }\end{array}$ & $15.96 \pm 17.28$ & $20.64 \pm 27.78$ & 22.7 \\
\hline
\end{tabular}

$I V$ Intravenous, $M M E$ morphine milligram equivalent

${ }^{a}$ Continuous variables are presented as the as mean $\pm \mathrm{SD}$

showed less optimized, lower mean VAS scores of $0-1$ for general pain at 72,96 , and $120 \mathrm{~h}$ postoperatively, which suggested potential over-administration of opioids. Whether this significantly lower VAS in the SOC versus the CryoNB cohort resulted in higher risk of somnolence and respiratory depression leading to lower FEV1 results is not known. This study was not designed nor powered to compare postoperative minimal analgesic effectiveness between the two study cohorts using VAS [23] as such, which remains an important topic for future investigation.

\section{Opioid Use}

Opioid-sparing pain management alternatives have been emphasized by both the Enhanced Recovery After Surgery Society guidelines (Class 1 Recommendation to pursue multimodal, opioid-sparing, pain management plan post cardiac surgery [27]) and a study using the STS Adult Cardiac Surgery Database, which identified that $12.5 \%$ and up to $15.7 \%$ of patients become newly persistent opioid users after cardiac and lung surgery, respectively [28]. Although the results of this study were not significant for opioid consumption, the trend was aligned with recent publications and demonstrated a $13 \%$ reduction in total opioid consumption in the CryoNB cohort compared to SOC (Table 5). Previous reports have suggested reduced opioid use after cryoanalgesia compared to conventional analgesia methods in thoracotomy procedures $[16,29]$. A recent systematic review of cryoanalgesia used in thoracic procedures concluded that intercostal cryoanalgesia may decrease inpatient narcotic use [30]. Therefore, future studies could be focused on further evaluating the impact of CryoNB on opioid requirements in minimally invasive cardiac procedures on cardiopulmonary bypass.

\section{Long-Term Allodynia}

In FROST, only 1 of the $63(1.6 \%)$ screened cryoNB patients tested positive using the cotton swab test for allodynia at 6 months [18]. At 10 months, the pain was not bothersome to a level that required pain management through medication. This rate is lower than that reported in a previous study comparing allodynia rates in patients who received cryoanalgesia or epidural analgesia [31]. Ju et al. reported significantly higher postoperative allodynia-like pain at 6 and 12 months with cryoanalgesia. In that study, allodynia was patient-reported through a questionnaire, whereas FROST required an in-office cotton tip applicator test for confirmation. Another recent study that used the same cryoprobe as FROST for cryo nerve block did not find any allodynia complications in treated patients after a median of 529 days (interquartile range 268-637 days); however the method of assessment was not specified in that study [29].

\section{Study Limitations}

This study had several limitations. Only $74 \%$ of the patients completed the 48 -h postoperative FEV1 assessment, with the primary reasons for 
patients being unable to perform this test being physical and physiological constraints. These constraints included being unable to get into optimal position to perform the test $(n=16)$, mortality $(n=3)$, prolonged oral intubation beyond $120 \mathrm{~h}(n=1)$, and hypoxia requiring high-flow oxygen delivered by nasal cannula $(n=1)$. We did not anticipate the difficulty that three consecutive efforts for FEV1 spirometry testing would pose for many of the patients recovering from $\mathrm{CPB}$ at $48 \mathrm{~h}$ postoperatively. In effect, patient compliance resulted in missing FEV1 values. Additionally, with no standardized study protocol for postoperative IV opioid administration, VAS assessment may have been confounded by the relative timing of administration of IV opioids (i.e., before or after opioid administration).

\section{CONCLUSIONS}

This study demonstrated that intercostal CryoNB in conjunction with SOC used in patients undergoing Mini-HVS requiring CPB provided a higher FEV1 recovery when compared to SOC alone. While similar therapeutic VAS scores were achieved at the surgical site between CryoNB versus SOC, CryoNB achieved an optimized VAS score for general pain versus SOC. A future randomized controlled trial is warranted to determine whether CryoNB has a specific opioid-sparing effect in this patient population.

\section{ACKNOWLEDGEMENTS}

We thank the participants of the study.

Funding. Sponsorship for this study and the journal's Rapid Service Fee were funded by AtriCure, Inc., Mason, OH, USA (ClinicalTrials.gov: NCT02922153).

Authorship. All named authors meet the International Committee of Medical Journal Editors (ICMJE) criteria for authorship for this article, take responsibility for the integrity of the work as a whole, and have given their approval for this version to be published.

Authors' Contributions. Conceptualization: W Lau; F Shannon, S Bolling, M Romano. Methodology: W Lau; F Shannon, S Bolling, M Romano, A Trescot. Formal analysis and investigation: all authors. Writing-original draft preparation: W Lau. Writing-review and editing: all authors.

Prior Presentation. This study was presented as an oral presentation at the annual Society for Thoracic Surgery meeting in New Orleans, LA, USA on 25 January 2020.

Disclosures. Wei Lau, Francis Shannon, Steven Bolling, Robert Johnson, and Vaughn Starnes declare that they have no conflicts of interest. Matthew Romano is a consultant for AtriCure and teaches training courses for surgical ablation. John Grehan is an educational and product development consultant for AtriCure. Andrea Trescot is a scientific consultant for AtriCure and Co-editor of Pain and Therapy. Luoxi Shi declares that she has no financial relationship with commercial interests. Marc Sawka is a consultant and speaker for Abbott. Dr. Sakwa changed institutions from Beaumont to Memorial Care during completion of the manuscript.

Compliance with Ethics Guidelines. The study was performed in accordance with the Helsinki Declaration of 1964 and subsequent amendments. Institutional review board (IRB) approval and written informed consent were obtained at all centers [University of Michigan, Ann Arbor, MI. (\#HUM00109220), University of Michigan Medical School IRB; Beaumont Health System, Royal Oak, MI (\#2015-438), Beaumont Research Institute IRB; United Heart and Vascular Institute-Allina, Saint Paul, MN (\#32157/1), Quorum IRB; and University of Southern California, Los Angeles, CA, University of Southern California Health Sciences Campus (IRB; \#HS-17-00066), University of Southern California Health Sciences Campus IRB]. 
Data Availability. The datasets generated during and/or analyzed during the current study are available at: https://clinicaltrials.gov/ ct2/show/NCT02922153.

Open Access. This article is licensed under a Creative Commons Attribution-NonCommercial 4.0 International License, which permits any non-commercial use, sharing, adaptation, distribution and reproduction in any medium or format, as long as you give appropriate credit to the original author(s) and the source, provide a link to the Creative Commons licence, and indicate if changes were made. The images or other third party material in this article are included in the article's Creative Commons licence, unless indicated otherwise in a credit line to the material. If material is not included in the article's Creative Commons licence and your intended use is not permitted by statutory regulation or exceeds the permitted use, you will need to obtain permission directly from the copyright holder. To view a copy of this licence, visit http://creativecommons.org/licenses/by$\mathrm{nc} / 4.0 /$.

\section{REFERENCES}

1. Kelsheimer B, Williams C, Kelsheimer C. New emerging modalities to treat post-thoracotomy pain syndrome: a review. Mo Med. 2019;116(1):41-4.

2. El-Hag-Aly MA, Hagag MG, Allam HK. If post-thoracotomy pain is the target, integrated thoracotomy is the choice. Gen Thorac Cardiovasc Surg. 2019;67(11):955-61.

3. Kearney DJ, Lee TH, Reilly JJ, DeCamp MM, Sugarbaker DJ. Assessment of operative risk in patients undergoing lung resection. Importance of predicted pulmonary function. Chest. 1994;105(3):753-9.

4. Berrisford R, Brunelli A, Rocco G, et al. The European Thoracic Surgery Database project: modelling the risk of in-hospital death following lung resection. Eur J Cardiothorac Surg. 2005;28(2):306-11.

5. Glauber M, Karimov JH, Farneti PA, et al. Minimally invasive mitral valve surgery via right minithoracotomy. Multimed Man Cardiothorac Surg. 2009;2009(122):mmcts.2008.003350.
6. Simpson BR, Parkhouse J, Marshall R, Lambrechts W. Extradural analgesia and the prevention of postoperative respiratory complications. $\mathrm{Br} \mathrm{J}$ Anaesth. 1961;33:628-41.

7. Chou R, Gordon DB, de Leon-Casasola OA, et al. Management of postoperative pain: a clinical practice guideline from the American Pain Society, the American Society of Regional Anesthesia and Pain Medicine, and the American Society of Anesthesiologists' Committee on Regional Anesthesia, Executive Committee, and Administrative Council. J Pain. 2016;17(2):131-57.

8. Jarzyna D, Jungquist CR, Pasero C, et al. American Society for Pain Management Nursing guidelines on monitoring for opioid-induced sedation and respiratory depression. Pain Manag Nurs. 2011;12(3): 118-45 e10.

9. Detterbeck FC. Efficacy of methods of intercostal nerve blockade for pain relief after thoracotomy. Ann Thorac Surg. 2005;80(4):1550-9.

10. Kang CM, Kim WJ, Yoon SH, Cho CB, Shim JS. Postoperative pain control by intercostal nerve block after augmentation mammoplasty. Aesthet Plast Surg. 2017;41(5):1031-6.

11. Joshi GP, Bonnet F, Shah R, et al. A systematic review of randomized trials evaluating regional techniques for postthoracotomy analgesia. Anesth Analg. 2008;107(3):1026-40.

12. Nishimori M, Ballantyne JC, Low JH. Epidural pain relief versus systemic opioid-based pain relief for abdominal aortic surgery. Cochrane Database Syst Rev. 2006;3:Cd005059.

13. Horlocker TT, Wedel DJ, Rowlingson JC, et al. Regional anesthesia in the patient receiving antithrombotic or thrombolytic therapy: American Society of Regional Anesthesia and Pain Medicine Evidence-Based Guidelines (third edition). Reg Anesth Pain Med. 2010;35(1):64-101.

14. Krishna SN, Chauhan S, Bhoi D, et al. Bilateral erector spinae plane block for acute post-surgical pain in adult cardiac surgical patients: a randomized controlled trial. J Cardiothorac Vasc Anesth. 2019;33(2):368-75.

15. Joucken K, Michel L, Schoevaerdts JC, Mayne A, Randour P. Cryoanalgesia for post-thoracotomy pain relief. Acta Anaesthesiol Belg. 1987;38(2): 179-83.

16. Moorjani N, Zhao F, Tian Y, Liang C, Kaluba J, Maiwand MO. Effects of cryoanalgesia on postthoracotomy pain and on the structure of intercostal nerves: a human prospective randomized 
trial and a histological study. Eur J Cardiothorac Surg. 2001;20(3):502-7.

17. Sepsas E, Misthos $\mathrm{P}$, Anagnostopulu M, Toparlaki O, Voyagis G, Kakaris $S$. The role of intercostal cryoanalgesia in post-thoracotomy analgesia. Interact Cardiovasc Thorac Surg. 2013;16(6):814-8.

18. Nasr-Esfahani M, Jarrell J. Cotton-tipped applicator test: validity and reliability in chronic pelvic pain. Am J Obstet Gynecol. 2013;208(1):52.e1-5.

19. Breivik H, Borchgrevink PC, Allen SM, et al. Assessment of pain. Br J Anaesth. 2008;101(1): 17-24.

20. Graham BL, Steenbruggen I, Miller MR, et al. Standardization of spirometry 2019 update. An official American Thoracic Society and European Respiratory Society technical statement. Am J Respir Crit Care Med. 2019;200(8):e70-88.

21. Gough JD, Williams AB, Vaughan RS, Khalil JF, Butchart EG. The control of post-thoracotomy pain. A comparative evaluation of thoracic epidural fentanyl infusions and cryo-analgesia. Anaesthesia. 1988;43(9):780-3.

22. Gwak MS, Yang M, Hahm TS, Cho HS, Cho $\mathrm{CH}$, Song JG. Effect of cryoanalgesia combined with intravenous continuous analgesia in thoracotomy patients. J Korean Med Sci. 2004;19(1):74-8.

23. Geppetti P, Benemei S. Pain treatment with opioids: achieving the minimal effective and the minimal interacting dose. Clin Drug Investig. 2009;29(Suppl 1):3-16.

24. Tan WH, Yu J, Feaman S, et al. Opioid medication use in the surgical patient: an assessment of prescribing patterns and use. J Am Coll Surg. 2018;227(2):203-11.

25. Lee LA, Caplan RA, Stephens LS, et al. Postoperative opioid-induced respiratory depression: a closed claims analysis. Anesthesiology. 2015;122(3): 659-65.

26. Myles PS, Myles DB, Galagher W, et al. Measuring acute postoperative pain using the visual analog scale: the minimal clinically important difference and patient acceptable symptom state. $\mathrm{Br} \mathrm{J}$ Anaesth. 2017;118(3):424-9.

27. Kaye AD, Urman RD, Rappaport Y, et al. Multimodal analgesia as an essential part of enhanced recovery protocols in the ambulatory settings. J Anaesthesiol Clin Pharmacol. 2019;35(Suppl 1): S40-5.

28. Brescia AA, Waljee JF, Hu HM, et al. Impact of prescribing on new persistent opioid use after cardiothoracic surgery. Ann Thorac Surg. 2019;108(4): 1107-13.

29. Tanaka A, Al-Rstum Z, Leonard SD, et al. Intraoperative intercostal nerve cryoanalgesia improves pain control post-descending, TAAA repairs. Ann Thorac Surg. 2019;109:249-54.

30. Cha PI, Min JG, Patil A, Choi J, Kothary NN, Forrester JD. Efficacy of intercostal cryoneurolysis as an analgesic adjunct for chest wall pain after surgery or trauma: systematic review. Trauma Surg Acute Care Open. 2021;6(1):e000690.

31. Ju H, Feng Y, Yang BX, Wang J. Comparison of epidural analgesia and intercostal nerve cryoanalgesia for post-thoracotomy pain control. Eur J Pain. 2008;12(3):378-84. 